\section{ESTRESSE OCUPACIONAL NO TRABALHO EM ENFERMAGEM NO BRASIL: UMA REVISÃO INTEGRATIVA}

\author{
Occupational stress at work in nursing in Brazil: an integrative \\ review
}
Estrés laboral del trabajo de enfermería en Brasil: una revisión integrativa

\section{RESUMO}

Objetivo: Descrever os fatores desencadeantes do estresse ocupacional em profissionais da enfermagem, bem como os riscos relacionados com o desenvolvimento desse estresse. Métodos: Constitui uma revisão integrativa da literatura científica sobre fatores desencadeantes do estresse ocupacional em profissionais da enfermagem e quais patologias estão associadas. Resultados: O método de análise da revisão integrativa baseou-se na categorização das informações coletadas na amostra final dos artigos. Assim, foram interpretados e agrupados em três categorias para compreensão do fenômeno: 1) Sinais, sintomas e patologias associadas ao estresse ocupacional; 2) Relação do estresse ocupacional com a síndrome de burnout; 3) Risco de estresse ocupacional e área de atuação em enfermagem. Conclusão: O profissional de enfermagem está cada vez mais predisposto ao adoecimento ocupacional motivado por estresse no ambiente de trabalho. Se por um lado, a população necessita dos enfermeiros, por outro, os trabalhadores da enfermagem também precisam de condições organizacionais favoráveis para desenvolver o seu papel que é suma importância para todos que vivem em sociedade.

Descritores: Enfermagem; Estresse; Brasil.

\section{ABSTRACT}

Objective: To describe the triggering factors of occupational stress in nursing professionals as well as the risks associated with its development. Methods: The study constitutes an integrative review of the scientific literature on triggering factors of occupational stress in nursing professionals and which conditions they are associated with. Results: The integrative review analysis method was based on the categorization of the information collected in the final sample of articles. Thus, they were interpreted and grouped into three categories for understanding of the phenomenon: 1) Signs, symptoms and pathologic conditions associated with occupational stress; 2) Association between occupational stress and the burnout syndrome; 3) Risk of occupational stress and areas of nursing practice. Conclusion: The nursing professional is increasingly prone to occupational sickening motivated by stress in the workplace. On the one hand, the population needs nurses; on the other, the nursing workers also need favorable organizational conditions to develop their role, which is very important for all who live in society.

Descriptors: Nursing; Stress, Brazil.
Artigo de Revisão

\section{Iel Marciano de Moraes Filho ${ }^{(1)}$ Rogério José de Almeida ${ }^{(1)}$}

Recebido em: 20/04/2016

Revisado em: $13 / 05 / 2016$

Aceito em: 22/08/2016 


\section{RESUMEN}

Objetivo: Describir los factores desencadenantes del estrés laboral de profesionales de enfermería asi como los riesgos relacionados al desarrollo de dicho estrés. Métodos: Revisión integrativa de la literatura cientifica sobre los factores desencadenantes del estrés laboral de profesionales de enfermería y cuales las patologías asociadas a él. Resultados: El método de análisis de la revisión integrativa estuvo basado en la categorización de las informaciones recogidas de la muestra final de los artículos. De esa manera, fueron interpretados y agrupados en tres categorías para la comprensión del fenómeno: 1) Signos, sintomas y patologías asociadas al estrés laboral; 2) Relación del estrés laboral con el síndrome de burnout; 3) Riesgo del estrés laboral y área de actuación en enfermería. Conclusión: El profesional de enfermería está más predispuesto al padecimiento laboral motivado por el estrés en el ambiente de trabajo. Si por un lado, la población necesita de enfermeros, de otro lado, los trabajadores de enfermería también necesitan de condiciones de organización favorables para el desarrollo de su papel que es muy importante para todos los que viven en sociedad.

Descriptores: Enfermería; Agotamiento Profesional; Brasil.

\section{INTRODUÇÃO}

A Saúde do trabalhador se define como um conjunto de diversas atividades que objetivam, por meio de ações de vigilância tanto de caráter epidemiológica como sanitária, a promoção, proteção, recuperação até a reabilitação da saúde dos trabalhadores que foram acometidos a riscos e agravos oriundos das condições laborais ${ }^{(1)}$.

A Política Nacional de Saúde do trabalhador e da trabalhadora foi instituída pela Portaria $\mathrm{n}^{\circ} 1.823$, de 23 de agosto de $2012^{(2)}$. Tem por finalidade definir os princípios, diretrizes e estratégias a serem observadas de uma maneira holística com uma dimensão tripartite, objetivando a promoção e proteção da saúde dos trabalhadores, logo minimizando a morbimortalidade decorrente dos processos de desenvolvimento e produtivos ${ }^{(2)}$.

Em consonância com as definições e finalidades das atividades de labor citadas acima, enfatiza-se a enfermagem, que por vez, é uma área que detém um aparato singular de conhecimentos técnico e científico, pautado e reproduzido por um conjunto de práticas sociais, éticas e políticas que se exprime pelo ensino, pesquisa e atividades assistenciais ${ }^{(3)}$.

Apesar das leis que asseguram a conservação da integridade dos trabalhadores como já fora citado, os profissionais de enfermagem se constitui em um público que na maioria das vezes cuidam de outras pessoas e se esquecem de si mesmo e do ambiente laboral. São profissionais que vêm a cada dia adoecendo, pelas condições insalubres de trabalho e ambientes desfavoráveis para o desenvolvimento de suas práticas ${ }^{(4)}$.

Em sua prática diária esses profissionais ao prestarem assistência direta ao paciente estão expostos a vários riscos correlacionados à atividade laboral que podem ser acometidos por fatores intrínsecos e extrínsecos como os riscos: químicos, físicos, mecânicos, biológicos e ergonômicos que podem causar agravos ocupacionais ${ }^{(4)}$. Esses profissionais também estão expostos ao enfrentar fatores que lhe causam demandas emocionais, diretamente ligados ao sofrimento dos pacientes e familiares que estão sendo assistidos ${ }^{(5)}$.

Um grande determinante de todas essas causas e o desenvolvimento de diversas patologias é o estresse. Esse agravo se caracteriza como um momento de tensão que causa desequilíbrio no funcionamento dos organismos, decorrentes de situações consideradas novas ou de risco e que ultrapassam há capacidade adaptativa do ser humano ${ }^{(6)}$.

O estresse também pode ser associado a outros agravos como a ansiedade, depressão e síndrome de burnout. A insatisfação pelo ambiente de trabalho, a condição socioeconômica dos trabalhadores, a falta de controle perante o trabalho e o baixo apoio e reconhecimento social de suas práticas figuram como fatores determinantes para o aparecimento do estresse ${ }^{(7)}$.

Os trabalhadores da enfermagem em sua prática diária prestam assistência direta ao paciente e estão expostos a vários riscos correlacionados à atividade laboral, por consequência, podem ser acometidos por diversas patologias, dentre elas, o estresse ocupacional. Nesse sentido, a presente revisão parte do seguinte problema de pesquisa: Quais os fatores associados e os riscos do desenvolvimento do estresse ocupacional em enfermeiros no Brasil?

Assim, a partir de uma revisão integrativa da literatura científica, o presente estudo tem por objetivo descrever os fatores desencadeantes do estresse ocupacional em profissionais da enfermagem, bem como os riscos relacionados com o desenvolvimento desse estresse.

\section{MÉTODOS}

Este estudo constitui uma revisão integrativa da literatura científica sobre fatores desencadeantes do estresse ocupacional em profissionais da enfermagem e quais patologias estão associadas. Para o alcance do objetivo proposto, optou-se por este tipo de revisão de literatura, uma vez que ela exibe a síntese de múltiplos estudos $\operatorname{científicos}^{(8)}$. Além disso, permite conclusões gerais de determinada área de estudo, corroborando para o aprofundamento e propagação do conhecimento dos problemas analisados 
em questão e de como ele tem sido estudado nas pesquisas atuais $^{(8)}$.

A revisão integrativa é um estudo que se dá a partir de a análise de pesquisas relevantes de fontes secundárias por meio de levantamento bibliográfico que reúne conhecimentos sobre o fenômeno a ser investigado. Constitui uma técnica de pesquisa com rigor metodológico, criteriosa e conscienciosa, que aumenta a credibilidade e a profundidade de conclusões que podem contribuir para reflexão sobre a realização de futuros estudos, desta forma contribuindo também para tomada de decisão que busque melhorar as evidências recentes ${ }^{(8)}$.

No presente estudo, optou-se por pesquisar em periódicos de divulgação científica em que foram consultadas as seguintes bases de dados: Biblioteca Virtual em Saúde (BVS), os Periódicos Capes e a United State National Library of Medicine (PubMed).

$\mathrm{Na}$ busca eletrônica dos artigos científicos e indexados nas bases de dados citadas, utilizaram-se os seguintes Descritores da Ciência da Saúde (DeCS) e suas combinações nas línguas inglesa e portuguesa: Enfermagem (Nursing), Stress (Estresse) e Brasil (Brazil).

Os seguintes critérios de inclusão para selecionar os estudos foram utilizados: indexação de estudos nas respectivas bases de dados; relação direta com os descritores; pesquisas desenvolvidas no Brasil; idiomas de publicação em português e inglês; período de publicação compreendido entre 2010 e 2015; estudos com pesquisa de campo e artigos disponíveis online. Foram excluídas deste estudo a revisões de literatura, dissertações, teses e editoriais. A coleta de dados foi realizada seguindo os critérios de inclusão no mês de outubro de 2015.

Ao se associarem os descritores foram encontradas 254 referências na BVS, 60 artigos no PubMed e 24 nos Periódicos Capes. Após a aplicação dos critérios de inclusão restaram 129 (BVS), 25 (PubMed) e 11 (Capes).

$\mathrm{Na}$ primeira fase, foi realizada a leitura dos títulos dos artigos e seus resumos. Após essa análise, foram selecionados 21 artigos da BVS, 6 dos Periódicos da Capes e 8 artigos da PubMed. Na Comparação dos resultados das buscas entre as bases de dados, do total de 35 artigos, 5 se repetiram restando assim 31 publicações selecionadas para leitura integral dos textos.

Na segunda fase, foi feita a leitura completa dos artigos, sendo excluídas as publicações que, embora contemplassem os descritores, não tratavam diretamente do tema objeto de estudo desta pesquisa. Após essa fase, foram selecionados 22 artigos que compõem a amostra final para o presente estudo.

Na terceira e última fase, os 22 artigos foram analisados a partir da identificação de categorias e variáveis com base no objetivo do presente estudo. Os conceitos-chave foram destacados e a análise seguiu o proposto na literatura específica da revisão integrativa de literatura ${ }^{(8)}$.

\section{RESULTADOS}

Os artigos referentes à temática de estresse ocupacional de enfermeiros no Brasil apresentaram variação nos anos de publicação, com período de maior e menor interesse pela temática. No ano de 2015 foram encontradas somente duas publicações $^{(9,10)}$, quatro em 2014 ${ }^{(11-14)}$, cinco em $2013^{(15-}$ ${ }^{19)}$, duas em 2012 (20,21), cinco em 2011 (22-26) e quatro em $2010^{(5,27-29)}$.

Os estudos se caracterizam com um percentual de abordagens metodológicas de 72,7\% (16) de estudos de caráter quantitativo, $22,7 \%$ (5) de qualitativos e $4,6 \%$ (1) que utilizaram as duas abordagens.

O quadro I descreve o conjunto dos 22 artigos selecionados neste estudo. Há uma descrição dos estudos incluídos nesta revisão integrativa, segundo autores, periódicos, ano de publicação e tipo de estudo.

\section{DISCUSSÃO}

O método de análise da revisão integrativa baseou-se na categorização das informações coletas na amostra final dos artigos. Assim, foram interpretados e agrupados em três categorias para compreensão do fenômeno: 1) Sinais, sintomas e patologias associadas ao estresse ocupacional; 2) Relação do estresse ocupacional com a Síndrome de Burnout; 3) Risco de estresse ocupacional e área de atuação em enfermagem.

\section{Sinais, sintomas e patologias associadas ao estresse ocupacional}

O estresse ocupacional pode ser causa do desenvolvimento de variadas doenças ou estar associado a outras patologias que determinam o absenteísmo da profissional de enfermagem causando prejuízo tanto para o trabalhador quanto para o empregador ${ }^{(9)}$.

A enfermagem é uma profissão de predominância feminina e, além dos desgastes advindos da complexidade da profissão, deve se associar a carga laboral extra da mulher dentro de seu domicílio e sua representação familiar. Muitas das vezes ela é a provedora e coordenadora das questões familiares, considerado também mais uma jornada de trabalho. São fatores que cooperam diretamente na qualidade e vida, na queda do rendimento, precariedade da assistência de enfermagem prestada a terceiros e insatisfação laboral, levando a disseminação de sinais, sintomas e patologias advindas do estresse ocupacional ${ }^{(17)}$. 
Quadro I - Caracterização dos artigos da amostra final, por ordem decrescente do ano de publicação. Goiânia, 2015.

\begin{tabular}{|c|c|c|}
\hline Autores & Periódico / Ano de publicação & Tipo de Estudo / Instrumento \\
\hline Silva et $\mathrm{al}^{(9)}$ & Rev. Bras. Ter. Intensiva (2015) & $\begin{array}{l}\text { Estudo quantitativo descritivo seccional utilizando } \\
\text { Maslach Burnout Inventory e o Self Report Questionnaire }\end{array}$ \\
\hline Zavalis et $\mathrm{al}^{(10)}$ & $\begin{array}{l}\text { Revista de Pesquisa: Cuidado é Fundamental } \\
\text { Online (2015) }\end{array}$ & $\begin{array}{l}\text { Estudo quantitativo correlacional descritivo, utilizando } \\
\text { questionário não validado }\end{array}$ \\
\hline Dalmolin et $\mathrm{al}^{(11)}$ & Revista Latino Americana de Enfermagem (2014) & $\begin{array}{l}\text { Estudo quantitativo, pesquisa de survey, utilizando } \\
\text { adaptação de Moral Distrees Scale (MDS) e de Masiach } \\
\text { Burnout Inventory (MBI) }\end{array}$ \\
\hline Oliveira et $\mathrm{al}^{(12)}$ & Revista de Enfermagem da UFRJ (2014) & $\begin{array}{l}\text { Estudo quantitativo utilizando Questionário Relação de } \\
\text { Ansiolíticos e trabalho (QRTCAT) QRCAT) elaborado } \\
\text { com base no AUDIT }\end{array}$ \\
\hline Ramos et $\mathrm{al}^{(13)}$ & $\begin{array}{l}\text { Revista de Pesquisa: Cuidado é Fundamental } \\
\text { Online (2014) }\end{array}$ & Pesquisa qualitativa descritiva \\
\hline Prochnow et $\mathrm{al}^{(14)}$ & Revista Latino Americana de Enfermagem(2014) & $\begin{array}{l}\text { Estudo epidemiológico transversal com abordagem } \\
\text { quantitativa utilizando Job Stress Scale (JSS) }\end{array}$ \\
\hline Oliveira et al ${ }^{(15)}$ & Revista de Enfermagem da UFRJ (2013) & $\begin{array}{l}\text { Pesquisa quantitativa Questionário Relação de Consumo } \\
\text { com o Trabalho (QRCAT) elaborado com base no AUDIT }\end{array}$ \\
\hline Oliveira et $\mathrm{al}^{(16)}$ & Revista de Enfermagem da UFRJ (2013) & Pesquisa qualitativa com roteiros de entrevista \\
\hline Oliveira et $\mathrm{al}^{(17)}$ & Revista da Escola de Enfermagem da USP (2013) & $\begin{array}{l}\text { Estudo exploratório e descritivo com abordagem } \\
\text { qualitativa }\end{array}$ \\
\hline Inoue et $\mathrm{al}^{(18)}$ & Revista Brasileira de Enfermagem (2013) & Estudo analítico transversal com abordagem quantitativa \\
\hline Lima et $\mathrm{al}^{(19)}$ & $\begin{array}{l}\text { Revista de Pesquisa: Cuidado é Fundamental } \\
\text { Online (2013) }\end{array}$ & $\begin{array}{l}\text { Estudo descritivo com abordagem quantitativa utilizou } \\
\text { questionário semiestruturado }\end{array}$ \\
\hline Fernandes et al $\mathrm{l}^{(20)}$ & $\begin{array}{l}\text { Revista de Pesquisa: Cuidado é Fundamental } \\
\text { Online (2012) }\end{array}$ & $\begin{array}{l}\text { Estudo descritivo com abordagem quantitativa utilizando } \\
\text { o Maslach Burnot Inventary (MBI) }\end{array}$ \\
\hline Maia et $\mathrm{al}^{(21)}$ & $\begin{array}{l}\text { Revista de Pesquisa: Cuidado é Fundamental } \\
\text { Online (2012) }\end{array}$ & $\begin{array}{l}\text { Pesquisa exploratória transversal de natureza quantitativa } \\
\text { utilizando Inventário de Sintomas de Stress para Adultos } \\
\text { de Lipp (ISSL) }\end{array}$ \\
\hline Farias et $\mathrm{al}^{(22)}$ & Revista da Escola de Enfermagem da USP (2011) & $\begin{array}{l}\text { Estudo quantitativo/qualitativo utilizou o Occupational } \\
\text { Stress Indicator (OS) e questionário semiestruturado }\end{array}$ \\
\hline Mininel et $\mathrm{al}^{(23)}$ & Revista Latino Americana de Enfermagem (2011) & Estudo descritivo com recorte qualitativo \\
\hline Griep et $\mathrm{al}^{(24)}$ & Revista de Saúde Pública (2011) & $\begin{array}{l}\text { Estudo transversal com abordagem quantitativa utilizando } \\
\text { questionário multidimensional }\end{array}$ \\
\hline Silva et $\mathrm{al}^{(25)}$ & Revista de Saúde Pública (2011) & $\begin{array}{l}\text { Estudo transversal com abordagem quantitativa, utilizou } \\
\text { versões adaptadas da Short Version of Job Stress Scale, } \\
\text { do Effort Reward Imbalance, SF } 36 \text { e do Work Ability } \\
\text { Index (Índice de Capacidade para o Trabalho - ICT). }\end{array}$ \\
\hline Linch et $\mathrm{al}^{(26)}$ & Revista Gaúcha de Enfermagem (2011) & $\begin{array}{l}\text { Estudo transversal com abordagem quantitativa utilizando } \\
\text { a Escala Estressora e Escala de Sintomas Apresentados } \\
\text { pelos Enfermeiros }\end{array}$ \\
\hline Dalri et $\mathrm{al}^{(5)}$ & Revista Ciência y Enfermería (2010) & $\begin{array}{l}\text { Estudo não experimental, com análise quantitativa de } \\
\text { dados utilizou questionário Codebook }\end{array}$ \\
\hline Rocha et $\mathrm{al}^{(27)}$ & Revista da Escola de Enfermagem da USP (2010) & $\begin{array}{l}\text { Estudo quantitativo transversal, descritivo e comparativo } \\
\text { utilizou a Escala de Bianchi de Stress Modificado - } \\
\text { EBSm e Questionário Índice de Qualidade do sono de } \\
\text { Pittisburgh-PSI }\end{array}$ \\
\hline Secco et $\mathrm{al}^{(28)}$ & $\begin{array}{l}\text { SMAD. Revista Eletrônica Saúde Mental Álcool e } \\
\text { Outras Drogas (2010) }\end{array}$ & $\begin{array}{l}\text { Estudo com abordagem qualitativa com técnica de análise } \\
\text { de conteúdo }\end{array}$ \\
\hline Feliciano et $\mathrm{al}^{(29)}$ & Revista de Saúde Pública (2010) & $\begin{array}{l}\text { Pesquisa com abordagem qualitativa utilizou entrevistas } \\
\text { com roteiro semiestruturado }\end{array}$ \\
\hline
\end{tabular}


Em síntese, os estudos apontam uma variedade de sinais e sintomas e patologias ocasionadas pelo estresse ocupacional entre eles estão: a) Sinais e Sintomas: Cefaleia, mialgia seguida com sensação de fadiga, sensação de desanimo pela manhã, dificuldades para dormir ou sono entrecortado, indisposição gástrica, e dores estomacais, taquicardia, tremores musculares, inapetência, sensação de folego curto e rubor facial, sensações de agulhadas pelo corpo, desgastes físicos, conflitos emocionais, cansaço, alterações cardiovasculares, lombalgias, síndromes depressivas, síndrome do pânico, esgotamento emocional, palpitações, extremidades frias, resfriados constantes, confusão, perda do senso de humor, raiva, frustação, preocupação, medo, irritabilidade, impaciência, náuseas em escala moderada, impotência, frustação, desconforto visual e ansiedade ${ }^{(5,9-14,16-29)}$. b) Patologias: Infarto agudo do miocárdio, distúrbios mentais neurológicos, psiquiátricos, síndromes depressivas, síndrome do pânico, hipertensão, gastrite, doenças somáticas e síndrome de burnout ${ }^{(5,9-11,13,14,16-29)}$.

Diante de todos estes agravos associados, seja por causa ou efeito do estresse ocupacional, o organismo do profissional de enfermagem necessita mobilizar energia extra para retomar seu equilíbrio inicial ${ }^{(19)}$.

\section{Relação do estresse ocupacional com a síndrome de burnout}

Alguns estudos demostraram a Síndrome de Burnout como sendo uma recorrente patologia associada e com prevalência dentre os agravos que podem ser desencadeados e ou como efeito do estresse ocupacional ${ }^{(9,11,19-21,29)}$.

Essa síndrome se caracteriza por três dimensões, sendo a exaustão emocional, a realização profissional e a despolarização. Uma pesquisa com 375 trabalhadores de enfermagem mostrou correlação entre sofrimento moral e a síndrome de burnout como potencial fonte de desenvolvimento da doença ${ }^{(11)}$.

O sofrimento moral está diretamente ligado ao desenvolvimento da síndrome de burnout, sendo que esta pode ocorrer por diversos fatores, dentre eles se destacam as práticas assistênciais e o envolvimento exacerbado do profissional da equipe de enfermagem com o cuidar ${ }^{(11)}$. Na maioria das vezes se desenvolve num período de 10 anos de exposição direta as atividades laborais associadas aos problemas cotidianos, tais como, trânsito e relacionamentos entre pacientes e cuidadores ${ }^{(9,21)}$.

Muitas vezes a equipe de enfermagem transcende o seu papel de cuidadora e passa a atribuir os sofrimentos e as problemáticas vivenciada pelos pacientes que estão sendo assistidos para si, querendo realizar o papel de advogado do paciente, acarretando ao profissional da equipe de enfermagem um estresse na defesa da integridade e dos valores do cuidado ${ }^{(11)}$.

Outro fator determinante em que a equipe de enfermagem tem dificuldade é no compartilhamento de saberes. A participação na equipe multiprofissional pode ficar deficitária acarretando conflitos, estresse e muitas vezes não conseguem ser membros efetivos de uma equipe multiprofissional com ênfase na interdisciplinaridade ${ }^{(19,29)}$.

A forte demanda de trabalho e a pressão exercida sobre o enfermeiro pelos gestores e pelos clientes na resolutividade dos atendimentos, além disso, o enorme investimento cognitivo e emocional que se dá na assistência direta prestada ao paciente são fatores que contribuem em larga escala para o desencadeamento da síndrome de burnout ${ }^{(19,29)}$.

$\mathrm{Na}$ maioria das vezes, o trabalhador insatisfeito consigo com características depressivas e irritantes próprias não consegue responder as próprias exigências, devido ao seu relacionamento intrapessoal estar colidente. Tais características podem gerar conflitos com a chefia, com a equipe e o afastamento do profissional de sua clientela como uma forma de refúgio ${ }^{(20)}$.

\section{Risco de estresse ocupacional e área de atuação em enfermagem}

Os estudos identificaram que a assistência de enfermagem compreendida nos serviços de alta e média complexidade tais como, atendimento pré-hospitalar (APH), pronto atendimento (PA), unidade de terapia intensiva (UTI), unidades públicas de urgência e emergência, pronto socorro (PS), unidades de hemodinâmica e os profissionais da área de enfermagem capacitados para atuarem nesses serviços, tais como os de socorristas e intesivistas relataram um maior grau de prevalência de estresse ocupacional ${ }^{(5,9,13,17,18,21,22,26,28)}$.

Observou-se que o ambiente de trabalho, de característica de alta e média complexidade, é considerado estressante. Nesses ambientes, os profissionais sofrem intensas demandas devido aos atendimentos de alta complexidade, que requerem elevadas cargas de prontidão e de responsabilidade, acarretando um desgaste emocional além dos problemas advindos da prática laboral do cuidar ${ }^{(21)}$.

Os estressores identificados em trabalhadores de enfermagem de alta e média complexidade compreendem paramentos altos de estresse ocupacional, salientando as alterações músculos esqueléticas ressaltadas pelos enfermeiros em estudo, corroborando aos fatores mentais relacionados ao estresse ocupacional( ${ }^{(26)}$.

Como fatores predisponentes do estresse ocupacional, independente do serviço em que os enfermeiros atuavam, os estudos mostraram uma insatisfação com prática laboral e relatam, por exemplo, uma carga horária que ultrapassa às 44 horas semanais podendo chegar até 160 horas. Associa- 
se a isso, a baixa remuneração dos trabalhadores e a atuação em outro vínculo empregatício na área de enfermagem para a complementação da renda mensal ${ }^{(5,9-14,16-20,22-29)}$.

É fato que a enfermagem conta como uma rotina de trabalho desgastante, de uma potencialização de talentos, de uma carga horária inflexível, complexa e fragmentada e de baixos salários ${ }^{(11,13,20)}$. Outras características predisponentes do estresse ocupacional foram a grande demanda, a cobrança para a efetivação com agilidade dos atendimentos, o medo de perda do vínculo empregatício, aumento gradativo da carga horária, o trabalho em três turnos na área hospitalar, trabalho em escala de plantão ${ }^{(10,14,17,19,22,25)}$. Outro fator de é demanda de atividades e responsabilidades acarretadas aos enfermeiros generalistas ${ }^{(9,22,24,29)}$.

Também foi mencionado para os estresses ocupacionais do profissional da enfermagem a alta prevalência de privatizações das unidades hospitalares causando inseguranças quanto à instabilidade dos empregos, a diminuição dos salários e a precariedade dos ambientes ${ }^{(15,17)}$.

A redução da mão de obra e a diminuição dos insumos para a assistência é outro ponto importante a ser destacado, pois os empresários querem a maximização dos lucros e esquecem-se da saúde do trabalhador e a qualidade da assistência em enfermagem que os profissionais prestam a seus pacientes ${ }^{(23,27)}$.

O trabalho de enfermagem é considerado muitas das vezes gratificante aos seus executores e também fonte de prazer e bem-estar. A assistência ao paciente com finalidade de promoção à saúde das pessoas é considerada importante no âmbito assistencial e a seus executores. Mesmo assim estes fatos podem causar no trabalhador um grande fator estressor, devido a não valorização da profissão em sua plenitude total ${ }^{(5)}$.

A morosidade e a burocracia dentro dos serviços de saúde são fatores dificultosos para os profissionais de enfermagem. $\mathrm{O}$ enfermeiro, muita das vezes, fica preso a essas obrigações, deixando de lado a assistência ao paciente. Esse fato pode atrasar a resolutividade dos agravos e, por consequência, a queda da qualidade de sua assistência ${ }^{(17)}$. Lidam ainda com os conflitos de funções que geram instabilidade na equipe multiprofissional ${ }^{(26)}$. Há também o acúmulo de funções para enfermeiro, considerado um fator estressor uma vez que em sua prática diária se perde entre os serviços gerenciais e assistênciais ${ }^{(18)}$.

$\mathrm{O}$ estresse ocupacional compromete o estado físico e metal do trabalhador da área da enfermagem. Assim, pode apresentar dificuldades para compreender os fatos que estão sendo vivenciados e de que forma esses estressores estão afetando a sua vida diária ${ }^{(13)}$.

Alguns estudos demonstraram que a incapacidade de enfrentamento dos problemas advindos do trabalho, na área da enfermagem, e o alto nível de estresse ocupacional podem levar os profissionais a dependência de álcool e outras drogas, e ao uso de ansiolíticos, na busca ativa para tentarem amenizar e remediar o sofrimento que estão expostos no dia a dia ${ }^{(12,15)}$. O consumo de álcool outras drogas preocupam, pois, o consumo excessivo como apresentado em uma pesquisa com 90 trabalhadores de enfermagem, resultou na ocorrência de $16,6 \%$ dos trabalhadores apresentando num intervalo de 30 dias, o uso regular e problemático de álcool causando dependência ${ }^{(15)}$.

\section{CONCLUSÃO}

Evidenciou-se que os fatores desencadeantes do estresse ocupacional estão relacionados à proximidade com o sofrimento humano, dificuldade do compartilhamento de saberes entre os membros da equipe multidisciplinar, carga horária elevada, demanda de trabalho, pressão exercida por gestores e pacientes, baixa remuneração, instabilidade do emprego, o trabalho desenvolvido em alta complexidade e o acúmulo de funções na prática diária.

O estresse ocupacional é inerente ao desenvolvimento de agravos ou associado a patologias que determinam o absenteísmo profissional. Dentre as doenças mais prevalentes, estão o infarto agudo do miocárdio, distúrbios mentais neurológicos, psiquiátricos, síndromes depressivas, síndrome do pânico, hipertensão, gastrite, doenças somáticas, síndrome de burnout, sendo esta última a mais recorrente. $\mathrm{O}$ estresse ocupacional também pode levar à dependência de álcool e outras drogas, bem como o uso constante de ansiolíticos.

\section{REFERÊNCIAS}

1. Brasil. Lei $\mathrm{n}^{0} 8080 / 90$. Dispõe sobre as condições para promoção, proteção e recuperação da saúde, a organização e o financiamento dos serviços correspondentes dá outras providências. Brasília: Ministério da Saúde; 1990.

2. Brasil. Portaria $n^{\circ} 1823 / 12$. Institui a Política Nacional de Saúde do Trabalhador e da Trabalhadora. Brasília: Ministério da Saúde; 2012.

3. Conselho Federal de Enfermagem (BR). Resolução Cofen 311/2007. Aprova a reformulação do Código de Ética dos Profissionais de Enfermagem. Rio de Janeiro: Cofen; 2007.

4. Ribeiro RP, Martins JT, Marziale MHP, Robazzi MLCC. $\mathrm{O}$ adoecer pelo trabalho na enfermagem: uma revisão integrativa. Rev Esc Enferm USP. 2012;46(2):495-504.

5. Dalri RCMB, Robazzi MLCC, Silva LA. Riscos ocupacionais e alterações de saúde entre trabalhadores 
de enfermagem brasileiros de unidades de urgência e emergência. Cienc Enferm. 2010;16(2):69-81.

6. KurebayashiLFS, Gnatta JR, Borges TP, Belisse G, Coca S, Minami A, et al. Aplicabilidade da auriculoterapia com agulhas ou sementes para diminuição de estresse em profissionais de enfermagem. Rev Esc Enferm USP. 2012;46(1):89-95.

7. Feitas AR, Carneseca EC, Paiva CE, Paiva BSR. Impacto de um programa de atividade física sobre a ansiedade, depressão, estresse ocupacional e síndrome de Burnout dos profissionais de enfermagem no trabalho. Rev Latinoam Enferm. 2014;22(2):332-6.

8. Mendes KDS, Silveira RCCP, Galvão CM. Revisão integrativa: método de pesquisa para a incorporação de evidências na saúde e na enfermagem. Texto \& Contexto Enferm. 2008;17(4):758-64.

9. Silva JLL, Soares RS, Costa FS, Ramos DS, Lima FB, Teixeira LR. Fatores psicossociais e prevalência da síndrome de burnout entre trabalhadores de enfermagem intensivistas. Rev Bras Ter Intensiva. 2015;27(2):125-33.

10. Zavalis A, Vianna LAM, Velasque LS, Schutz V, Machado DA. A influência dos fatores estressores sobre os níveis de atenção de profissionais de enfermagem. Rev Pesqui Cuid Fundam (Online). 2015;7(4):337587.

11. Dalmolin GL, Lunardi VL, Lunardi GL, Barlem ELD, Silveira RS. Sofrimento moral e síndrome de Burnout: existem relações entre esses fenômenos nos trabalhadores de enfermagem? Rev Latinoam Enferm. 2014;22(1):35-42.

12. Oliveira EB, Araújo PMB, Maia MPQ, Cabral JL, Brito DM, Figueredo EP. Estresse ocupacional e consumo de ansiolíticos por trabalhadores de enfermagem. Rev Enferm UERJ. 2014,22(5):615-21.

13. Ramos EL, Souza NVDO, Gonçalves FGA, Pires AS, Santos DM. Qualidade de vida no trabalho: repercussões para a saúde do trabalhador de enfermagem de terapia intensiva. Rev Pesqui Cuid Fundam (Online). 2014;6(2):571-83.

14. Prochnow A, Magnago TSBS, Urbanetto JS, Beck CLC, Lima SBS, Greco PBT. Capacidade para o trabalho na enfermagem: relação com demandas psicológicas e controle sobre o trabalho. Rev. Latinoam Enferm. 2013;21(6):1298-305.

15. Oliveira EB, Fabri JMG, Paula GS, Souza SRC, Silveira WC, Matos GS. Padrões de uso de álcool por trabalhadores de enfermagem e associação com trabalho. Rev Enferm UERJ. 2013;21(Esp 2):729-35.
16. Oliveira EB, Silva AV, Perez EF Júnior, Costa HF, Nascimento LP, Souza LAM. Fatores de risco psicossocial em terapia intensiva neonatal: repercussões para a saúde do enfermeiro. Rev Enferm UERJ. 2013;21(4):490-5.

17. Oliveira JDS, Achieri JC, Pessoa JM Júnior, Miranda FAN, Almeida MG. Representações sociais de enfermeiros acerca do estresse laboral em um serviço de urgência. Rev Esc Enferm USP. 2013;47(4):984-9.

18. Inoue KC, Versa GLGS, Murassaki ACY, Melo WA, Matsuda LM. Estresse ocupacional em enfermeiros intensivistas que prestam cuidados diretos ao paciente crítico. Rev Bras Enferm. 2013;66(5):722-9.

19. Lima MB, Silva LMS, Almeida FCM, Torres RAM, Dourado HHM. Agentes estressores em trabalhadores de enfermagem com dupla ou mais jornada de trabalho. Rev Pesqui Cuid Fundam (Online). 2013;5(1):3259-66.

20. Fernandes MA, Sousa FK, Santos JS, Rodrigues JA, Marziale MHP. Síndrome de Burnout em profissionais de enfermagem do serviço de atendimento móvel de urgência. Rev Pesqui Cuid Fundam (Online). 2012;4(4):3125-35.

21. Maia EC, Miranda MDC, Caetano JÁ, Carvalho ZMF, Santos MCL, Caldini LN. Avaliação do nível de estresse de equipe de enfermagem de serviço de atendimento móvel de urgência. Rev Pesqui Cuid Fundam (Online). 2012;4(4):3060-8.

22. Farias SMC, Teixeira OLC, Moreira W, Oliveira MAF, Pereira MO. Caracterização dos sintomas físicos de estresse na equipe de pronto atendimento. Rev Esc Enferm USP. 2011;45(3):722-9.

23. Mininel VA, Baptista PCP, Felli VEA. Cargas psíquicas e processos de desgaste em trabalhadores de enfermagem de hospitais universitários brasileiros. Rev Latinoam Enferm. 2011;19(2):340-7.

24. Griep RH, Rotenberg L, Landsbergis P, VasconcellosSilva PR. Uso combinado de modelos de estresse no trabalho e a saúde auto-referida na enfermagem. Rev Saúde Pública. 2011;45(1):145-52.

25. Silva AA, Rotengerg L, Fischer FM. Jornadas de trabalho na enfermagem: entre necessidades individuais e condições de trabalho. Rev Saúde Pública. 2011;45(6):1117-26.

26. Linch GFC, Guido LA. Estresse de enfermeiros em unidade de hemodinâmica no Rio Grande do Sul, Brasil. Rev Gaúcha Enferm. 2011;32(1):63-71.

27. Rocha MCP, Martino MMF. O estresse e qualidade de sono do enfermeiro nos diferentes turnos hospitalares. Rev Esc Enferm. USP. 2010;44(2):280-6. 
28. Secco IAO, Robazzi MLCC, Souza FEA, Shimizu DS. Cargas psíquicas de trabalho e desgaste dos trabalhadores de enfermagem de hospital de ensino do Paraná, Brasil. SMAD Rev Eletrônica Saúde Mental Álcool Drog. 2010;6(1):1-17.

29. Feliciano KVO, Kovacs MH, Sarinho SW. Superposição de atribuições e autonomia técnica entre enfermeiras da Estratégia Saúde da Família. Rev Saúde Pública. 2010;44(3):520-7.
Endereço do primeiro autor:

Iel Marciano de Moraes Filho

Pontifícia Universidade Católica de Goiás - PUC

Avenida Universitária, 1440

Bairro: Setor Universitário

CEP: 74605-010 - Goiânia - GO - Brasil

E-mail: ielfilho@ibest.com.br

Endereço para correspondência:

Rogério José de Almeida

Pontifícia Universidade Católica de Goiás - PUC

Avenida Universitária, 1440

Bairro: Setor Universitário

CEP: 74605-010 - Goiânia - GO - Brasil

E-mail: rogeriopucgo@gmail.com 